Copyright (C 2015 IEEE. Personal use of this material is permitted. Permission from IEEE must be obtained for all other uses, in any current or future media, including reprinting/republishing this material for advertising or promotional purposes, creating new collective works, for resale or redistribution to servers or lists, or reuse of any copyrighted component of this work in other works. 


\title{
Output Stabilization of Boundary-controlled Parabolic PDEs via Gradient-based Dynamic Optimization*
}

\author{
Zhigang Ren ${ }^{1}$, Chao $\mathrm{Xu}^{1}$, Qun $\mathrm{Lin}^{2}$, and Ryan Loxton ${ }^{2}$
}

\begin{abstract}
This paper proposes a new control synthesis approach for the stabilization of boundary-controlled parabolic partial differential equations (PDEs). In the proposed approach, the optimal boundary control is expressed in integral state feedback form with quadratic kernel function, where the quadratic's coefficients are decision variables to be optimized. We introduce a system cost functional to penalize both state and kernel magnitude, and then derive the cost functional's gradient in terms of the solution of an auxiliary "costate" PDE. On this basis, the output stabilization problem can be solved using gradient-based optimization techniques such as sequential quadratic programming. The resulting optimal boundary control is guaranteed to yield closed-loop stability under mild conditions. The primary advantage of our new approach is that the costate PDE is in standard form and can be solved easily using the finite difference method. In contrast, the traditional control synthesis approaches for boundary-controlled parabolic PDEs (i.e., the LQ control and backstepping approaches) require solving nonstandard Riccati-type and Klein-Gorden-type PDEs.
\end{abstract}

\section{INTRODUCTION}

Parabolic partial differential equation (PDE) systems are an important type of distributed parameter system (DPS) describing a wide range of natural phenomena, including diffusion, heat transfer, and fusion plasma transport. Over the past few decades, control theory for the parabolic DPS has developed into a mature research topic at the interface of engineering and applied mathematics [1], [2], [10].

The linear quadratic (LQ) control framework is welldefined in infinite dimensional function spaces to deal with the parabolic DPS (e.g., [1], [2]). However, the LQ control framework requires solving Riccati-type differential equations, which are nonlinear parabolic PDEs of dimension one greater than the original parabolic PDE system. For example, to generate an optimal feedback controller for a scalar heat equation, a Riccati PDE defined over a rectangular domain must be solved [11]. Hence, the LQ approach does not actually solve the controller synthesis problem directly, but instead converts it into another problem (i.e., solve a Riccati-type PDE) that is still extremely difficult to solve from a computational point of view.

*This work was supported by the National Natural Science Foundation of China (grants F030119-61104048 and 61320106009) and the National High Technology Research and Development Program of China (grant 2012AA041701)

${ }^{1} \mathrm{Z}$. Ren and C. Xu are with the State Key Laboratory of Industrial Control Technology and the Institute of Cyber-Systems \& Control, Zhejiang University, Hangzhou, China. renzhigang@zju.edu.cn, cxuezju. edu. cn

${ }^{2} \mathrm{Q}$. Lin and R. Loxton are with the Department of Mathematics \& Statistics, Curtin University, Perth, Australia. q. Iin@curtin.edu.au, r.loxtonecurtin.edu. au

Correspondence to: cxu@zju.edu.cn
One of the major advances in PDE control in recent years has been the so-called infinite dimensional Voltera integral feedback, or the backstepping method (e.g., [5], [9]). Instead of Riccati-type PDEs, the backstepping method requires solving the so-called kernel equations-linear Klein-Gordentype PDEs for which the successive approach can be used to obtain explicit solutions. This method was originally developed for the stabilization of one dimensional parabolic DPS and then extended to fluid flows [16], [19], magnetohydrodynamic flows [17], and elastic vibration [4]. In addition, the backstepping method can also be applied to achieve full state feedback stabilization and state estimation of PDE-ODE cascade systems [13].

In this paper, we propose a new framework for control synthesis for boundary-controlled parabolic DPS. This new framework does not require solving Riccati-type or KleinGorden-type PDEs. Instead, it requires solving a so-called "costate" PDE, which is much easier to solve from a computational viewpoint. In fact, many numerical software packages, such as Comsol Multiphysics and MATLAB PDE ToolBox, can be used to generate numerical solutions for the costate PDE. The Riccati PDEs, on the other hand, are usually not in standard form and thus cannot be solved using off-the-shelf software packages. The approach proposed in this paper can be viewed as an extension of optimization-based PID tuning ideas (see [3], [6], [14], [18]) to infinite dimensional systems.

\section{PROBLEM FORMULATION}

\section{A. Feedback Kernel Optimization}

We consider the following parabolic PDE system:

$$
\left\{\begin{array}{l}
y_{t}(x, t)=y_{x x}(x, t)+c y(x, t), \\
y(0, t)=0, \\
y(1, t)=u(t), \\
y(x, 0)=y_{0}(x),
\end{array}\right.
$$

where $c>0$ is a given constant and $u(t)$ is a boundary control. It is well known that the uncontrolled version of system (1) is unstable when the constant $c$ is sufficiently large [5]. According to the LQ control [11] and backstepping synthesis approaches [5], the optimal stabilizing control law takes the following feedback form:

$$
u(t)=\int_{0}^{1} \mathscr{K}(1, \xi) y(\xi, t) d \xi,
$$

where the feedback kernel $\mathscr{K}(1, \xi)$ is obtained by solving either a Riccati-type or a Klein-Gorden-type PDE. By introducing the new notation $k(\xi)=\mathscr{K}(1, \xi)$, we can write the 


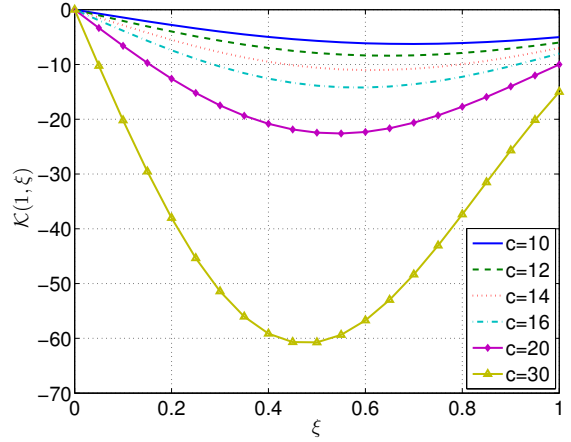

Fig. 1. The feedback kernel (4) for various values of $c$.

feedback control policy (2) in the following form:

$$
u(t)=\int_{0}^{1} k(\xi) y(\xi, t) d \xi .
$$

The corresponding closed-loop system is

$$
\left\{\begin{array}{l}
y_{t}(x, t)=y_{x x}(x, t)+c y(x, t), \\
y(x, 0)=y_{0}(x), \\
y(0, t)=0, \\
y(1, t)=\int_{0}^{1} k(\xi) y(\xi, t) d \xi .
\end{array}\right.
$$

In reference [5], the backstepping method is used to express the optimal feedback kernel as follows:

$$
\mathscr{K}(1, \xi)=-c \xi \frac{I_{1}\left(\sqrt{c\left(1-\xi^{2}\right)}\right)}{\sqrt{c\left(1-\xi^{2}\right)}},
$$

where $I_{1}$ is the first-order modified Bessel function given by

$$
I_{1}(\omega)=\sum_{n=0}^{\infty} \frac{\omega^{2 n+1}}{2^{2 n+1} n !(n+1) !} .
$$

The feedback kernel (4) is plotted in Figure 1 for different values of $c$. Note that its shape is similar to a quadratic function. Note also that $\mathscr{K}(1, \xi)=0$ when $\xi=0$. Accordingly, motivated by the quadratic behavior exhibited in Fig. 1, we express $k(\xi)$ in the following parameterized form:

$$
k(\xi ; \Theta)=\theta_{1} \xi+\theta_{2} \xi^{2},
$$

where $\Theta=\left(\theta_{1}, \theta_{2}\right)^{\top}$ is a parameter vector to be optimized.

Moreover, we assume that the parameters must satisfy the following bound constraints:

$$
a_{1} \leq \theta_{1} \leq b_{1}, \quad a_{2} \leq \theta_{2} \leq b_{2},
$$

where $a_{1}, a_{2}, b_{1}$ and $b_{2}$ are given bounds.

Let $y(x, t ; \Theta)$ denote the solution of the closed-loop system (3) with the parameterized kernel (5). The results in [15] ensure that such a solution exists and is unique. Our goal is to stabilize the closed-loop system with minimal energy input. Accordingly, we consider the following cost functional:

$$
g_{0}(\Theta)=\frac{1}{2} \int_{0}^{T} \int_{0}^{1} y^{2}(x, t ; \Theta) d x d t+\frac{1}{2} \int_{0}^{1} k^{2}(x ; \Theta) d x .
$$

We now state our kernel optimization problem formally as follows.

Problem 2.1: Given the PDE system (3) with the parameterized kernel (5), find an optimal parameter vector $\Theta=$ $\left(\theta_{1}, \theta_{2}\right)^{\top}$ such that the cost functional (7) is minimized subject to the bound constraints (6).

\section{B. Closed-Loop Stability}

Since (7) is a finite-time cost functional, there is no guarantee that the optimized kernel (5) generated by the solution of Problem 2.1 stabilizes the closed-loop system (3) as $t \rightarrow \infty$. Nevertheless, we now show that, by analyzing the solution structure of (3), additional constraints can be added to Problem 2.1 to ensure closed-loop stability.

Using the separation of variables approach, we decompose $y(x, t)$ as follows:

$$
y(x, t)=\mathscr{X}(x) \mathscr{T}(t) .
$$

Substituting (8) into (3a), we obtain

$$
\mathscr{X}(x) \dot{\mathscr{T}}(t)=\mathscr{X}^{\prime \prime}(x) \mathscr{T}(t)+c \mathscr{X}(x) \mathscr{T}(t),
$$

where

$$
\dot{\mathscr{T}}(t)=\frac{d \mathscr{T}(t)}{d t}, \quad \mathscr{X}^{\prime \prime}(x)=\frac{d^{2} \mathscr{X}(x)}{d x^{2}} .
$$

Furthermore, from the boundary conditions (3c) and (3d),

$$
\begin{aligned}
& \mathscr{X}(0) \mathscr{T}(t)=0, \\
& \mathscr{X}(1) \mathscr{T}(t)=\int_{0}^{1} k(\xi ; \Theta) \mathscr{X}(\xi) \mathscr{T}(t) d \xi .
\end{aligned}
$$

Thus, we immediately obtain

$$
\begin{gathered}
\mathscr{X}(0)=0, \\
\mathscr{X}(1)=\int_{0}^{1} k(\xi ; \Theta) \mathscr{X}(\xi) d \xi .
\end{gathered}
$$

Rearranging (9) gives

$$
\frac{\mathscr{X}^{\prime \prime}(x)+c \mathscr{X}(x)}{\mathscr{X}(x)}=\frac{\dot{\mathscr{T}}(t)}{\mathscr{T}(t)}=\sigma,
$$

where $\sigma$ is a constant called the eigenvalue. Clearly,

$$
\mathscr{T}(t)=T_{0} \mathrm{e}^{\sigma t}
$$

where $T_{0}=\mathscr{T}(0)$ is a constant to be determined.

To solve for $\mathscr{X}(x)$, we must consider three cases: (i) $c<\sigma$; (ii) $c=\sigma$; (iii) $c>\sigma$. In cases (i) and (ii), the general solutions of (12) are, respectively,

$$
\mathscr{X}(x)=X_{0} \mathrm{e}^{\sqrt{\sigma-c} x}+X_{1} \mathrm{e}^{-\sqrt{\sigma-c} x},
$$

and

$$
\mathscr{X}(x)=X_{0}+X_{1} x
$$

where $X_{0}$ and $X_{1}$ are constants to be determined from the boundary conditions (10) and (11). Then the corresponding solutions of (3) are

$$
y(x, t)=X_{0} T_{0} \mathrm{e}^{\sqrt{\sigma-c} x+\sigma t}+X_{1} T_{0} \mathrm{e}^{-\sqrt{\sigma-c} x+\sigma t},
$$


and

$$
y(x, t)=X_{0} T_{0} \mathrm{e}^{\sigma t}+X_{1} T_{0} x \mathrm{e}^{\sigma t} .
$$

These solutions are clearly unstable because $0<c \leq \sigma$. Thus, the parameters $\theta_{1}$ and $\theta_{2}$ should be chosen so that the unique solution of (3) satisfies case (iii) instead of cases (i) and (ii).

In case (iii), the general solution of (12) is

$$
\mathscr{X}(x)=X_{0} \cos (\sqrt{c-\sigma} x)+X_{1} \sin (\sqrt{c-\sigma} x),
$$

where $X_{0}$ and $X_{1}$ are constants to be determined from the boundary conditions (10) and (11). Substituting (14) into (10), we obtain

$$
\mathscr{X}(0)=X_{0}=0 .
$$

Hence,

$$
\mathscr{X}(x)=X_{1} \sin (\sqrt{c-\sigma} x) .
$$

To simplify the notation, we introduce a new variable $\alpha=$ $\sqrt{c-\sigma}$. Substituting (15) into condition (11), we have

$$
X_{1} \sin \alpha=X_{1} \int_{0}^{1} \theta_{1} \xi \sin (\alpha \xi) d \xi+X_{1} \int_{0}^{1} \theta_{2} \xi^{2} \sin (\alpha \xi) d \xi
$$

and thus

$$
\sin \alpha=\int_{0}^{1} \theta_{1} \xi \sin (\alpha \xi) d \xi+\int_{0}^{1} \theta_{2} \xi^{2} \sin (\alpha \xi) d \xi .
$$

By evaluating the integrals on the right-hand side, equation (16) can be simplified to obtain

$$
\begin{aligned}
& \left(\theta_{1} \alpha^{2}+\theta_{2} \alpha^{2}-2 \theta_{2}\right) \cos \alpha \\
& \quad+\left(\alpha^{3}-\theta_{1} \alpha-2 \theta_{2} \alpha\right) \sin \alpha+2 \theta_{2}=0 .
\end{aligned}
$$

For any $\alpha$ satisfying (17), there exists a corresponding solution of (12) in the form (15). It can be shown that (17) has an infinite number of positive solutions when $\Theta=\left(\theta_{1}, \theta_{2}\right)^{\top}$ satisfies the following inequality:

$$
\theta_{1}^{2}+\theta_{2}^{2}+2 \theta_{1} \theta_{2}-2 \theta_{1}-4 \theta_{2} \geq 0 .
$$

This is demonstrated numerically in Section IV. A formal proof will be given in a forthcoming journal article [12]. Let $\left\{\alpha_{n}\right\}_{n=1}^{\infty}$ be a sequence of positive solutions of (17). Then the general solution of (12) is

$$
\mathscr{X}(x)=\sum_{n=1}^{\infty} A_{n} \sin \left(\alpha_{n} x\right),
$$

where $A_{n}$ are constants to be determined. The corresponding eigenvalues are

$$
\sigma_{n}=c-\alpha_{n}^{2}, \quad n=1,2,3, \ldots
$$

Hence, using (13),

$$
y(x, t)=\mathscr{X}(x) \mathscr{T}(t)=\sum_{n=1}^{\infty} T_{0} A_{n} \mathrm{e}^{\left(c-\alpha_{n}^{2}\right) t} \sin \left(\alpha_{n} x\right) .
$$

By virtue of (10) and (11), this solution satisfies the boundary conditions (3c) and (3d). The constants $T_{0}$ and $A_{n}$ must be selected appropriately so that the initial condition (3b) is also satisfied. To ensure stability as $t \rightarrow \infty$, each eigenvalue $\sigma_{n}=$ $c-\alpha_{n}^{2}$ in (18) must be negative. Thus, we impose the following constraints on $\Theta=\left(\theta_{1}, \theta_{2}\right)^{\top}$ :

$$
\begin{aligned}
& \theta_{1}^{2}+\theta_{2}^{2}+2 \theta_{1} \theta_{2}-2 \theta_{1}-4 \theta_{2} \geq 0, \\
& c-\alpha^{2} \leq-\varepsilon, \\
& \left(\theta_{1} \alpha^{2}+\theta_{2} \alpha^{2}-2 \theta_{2}\right) \cos \alpha \\
& \quad+\left(\alpha^{3}-\theta_{1} \alpha-2 \theta_{2} \alpha\right) \sin \alpha+2 \theta_{2}=0,
\end{aligned}
$$

where $\varepsilon$ is a given positive parameter and $\alpha$ is the smallest positive solution of (17). Note that $\alpha$ here is treated as an additional optimization variable. Constraint (19a) ensures that there are an infinite number of eigenvalues and thus the solution form (18) is valid. Constraints (19b) and (19c) ensure that the largest eigenvalue is negative, thus guaranteeing solution stability. Adding constraints (19) to Problem 2.1 yields the following modified problem.

Problem 2.2: Given the PDE system (3) with the parameterized kernel (5), choose $\Theta=\left(\theta_{1}, \theta_{2}\right)^{\top}$ and $\alpha$ such that the cost functional (7) is minimized subject to the bound constraints (6) and the nonlinear constraints (19).

The next result is concerned with the stability of the closedloop system corresponding to the optimized kernel from Problem 2.2.

Theorem 2.1: Let $\left(\Theta^{*}, \alpha^{*}\right)$ be an optimal solution of Problem 2.2, where $\alpha^{*}$ is the smallest positive solution of equation $(19 \mathrm{c})$ corresponding to $\Theta^{*}$. Suppose that there exists a sequence $\left\{\alpha_{n}^{*}\right\}_{n=1}^{\infty}$ of positive solutions to equation (19c) corresponding to $\Theta^{*}$ such that $y_{0}(x) \in \operatorname{span}\left\{\sin \left(\alpha_{n}^{*} x\right)\right\}$. Then the closed-loop system (3) corresponding to $\Theta^{*}$ is stable.

Proof: Because of constraint (19a), the solution form (18) with $\alpha_{n}=\alpha_{n}^{*}$ is guaranteed to satisfy (3a), (3c) and (3d). If $y_{0}(x) \in \operatorname{span}\left\{\sin \left(\alpha_{n}^{*} x\right)\right\}$, then there exists constants $Y_{n}, n \geq 1$, such that

$$
y_{0}(x)=\sum_{n=1}^{\infty} Y_{n} \sin \left(\alpha_{n}^{*} x\right) .
$$

Taking $Y_{n}=T_{0} A_{n}$ ensures that (18) with $\alpha_{n}=\alpha_{n}^{*}$ also satisfies the initial conditions ( $3 b)$, and is therefore the unique solution of (3). Since $\alpha^{*}$ is the first positive solution of equation (17), it follows from constraints (19b) and (19c) that for each $n \geq 1$,

$$
c-\left(\alpha_{n}^{*}\right)^{2} \leq c-\left(\alpha^{*}\right)^{2} \leq-\varepsilon<0 .
$$

This shows that all eigenvalues are negative.

Theorem 2.1 requires that the initial function $y_{0}(x)$ be contained within the linear span of sinusoidal functions $\sin \left(\alpha_{n}^{*} x\right)$, where each $\alpha_{n}^{*}$ is a solution of equation (17) corresponding to $\Theta^{*}$. The good thing about this condition is that it can be verified numerically by solving the following optimization problem: choose span coefficients $Y_{n}, 1 \leq n \leq N$, to minimize

$$
J=\int_{0}^{1}\left|y_{0}(x)-\sum_{n=1}^{N} Y_{n} \sin \left(\alpha_{n}^{*} x\right)\right|^{2} d x,
$$

where $N$ is a sufficiently large integer and each $\alpha_{n}^{*}$ is a solution of equation (17) corresponding to the optimal solution of Problem 2.2. If the optimal cost value for this optimization 
problem is sufficiently small, then the span condition in Theorem 2.1 is likely to be satisfied, and therefore closed-loop stability is expected. Based on our computational experience, the span condition in Theorem 2.1 is usually satisfied. In fact, as we show in [12], the solutions $\alpha_{n}^{*}$ of (17) converge to the integer multiples of $\pi$. Thus, it is reasonable to expect that the linear span of $\left\{\sin \left(\alpha_{n}^{*} x\right)\right\}$ is "approximately" the same as the linear span of $\{\sin (n \pi x)\}$, which is known to be a basis for the space of continuous functions defined on $[0,1]$.

\section{NUMERICAL COMPUTATION}

Problem 2.2 is an optimal parameter selection problem with decision parameters $\theta_{1}, \theta_{2}$ and $\alpha$. In principle, such problems can be solved as nonlinear optimization problems using sequential quadratic programming or other nonlinear optimization methods. However, to do this, we need the gradients of the cost functional (7) and the constraint functions (19) with respect to the decision parameters.

Since the constraint functions in (19) are explicit functions of the decision variables, their gradients are easily derived using elementary differentiation. The cost functional (7), on the other hand, is an implicit function of $\Theta$ because it depends on the state trajectory $y(x, t)$. Thus, computing the gradient of (7) is a non-trivial task. We now develop a computational method, analogous to the costate method in the optimal control of ordinary differential equations [7], [8], [14], for computing this gradient.

We define the following costate PDE system:

$$
\left\{\begin{array}{l}
v_{t}(x, t)+v_{x x}(x, t)+c v(x, t) \\
\quad+y(x, t ; \Theta)-k(x ; \Theta) v_{x}(1, t)=0, \\
v(0, t)=v(1, t)=0, \\
v(x, T)=0 .
\end{array}\right.
$$

Let $v(x, t ; \Theta)$ denote the solution of the costate PDE system (21) corresponding to the parameter vector $\Theta$. Then we have the following theorem.

Theorem 3.1: The gradient of the cost functional (7) is given by

$$
\begin{aligned}
& \nabla_{\theta_{1}} g_{0}(\Theta)=-\int_{0}^{T} \int_{0}^{1} x v_{x}(1, t) y(x, t) d x d t+\frac{1}{3} \theta_{1}+\frac{1}{4} \theta_{2}, \\
& \nabla_{\theta_{2}} g_{0}(\Theta)=-\int_{0}^{T} \int_{0}^{1} x^{2} v_{x}(1, t) y(x, t) d x d t+\frac{1}{4} \theta_{1}+\frac{1}{5} \theta_{2},
\end{aligned}
$$

where $y(x, t)=y(x, t ; \Theta)$ and $v_{x}(x, t)=v_{x}(x, t ; \Theta)$.

Proof: For simplicity, we write $y(x, t ; \Theta)$ as $y(x, t)$ and $k(x ; \Theta)$ as $k(x)$. Let $\psi(x, t)$ be an arbitrary function satisfying

$$
\psi(x, T)=0, \quad \psi(0, t)=\psi(1, t)=0 .
$$

Then we can rewrite the cost functional (7) in augmented form as follows:

$$
\begin{aligned}
& g_{0}(\Theta)=\frac{1}{2} \int_{0}^{T} \int_{0}^{1} y^{2}(x, t) d x d t+\frac{1}{2} \int_{0}^{1} k^{2}(x) d x \\
& +\int_{0}^{T} \int_{0}^{1} \psi(x, t)\left\{-y_{t}(x, t)+y_{x x}(x, t)+c y(x, t)\right\} d x d t .
\end{aligned}
$$

Using integration by parts and applying conditions (3b), (3c) and (22), we can simplify the augmented cost functional (23) to obtain

$$
\begin{aligned}
g_{0}(\Theta)= & \frac{1}{2} \int_{0}^{T} \int_{0}^{1} y^{2}(x, t) d x d t+\frac{1}{2} \int_{0}^{1} k^{2}(x) d x \\
& +\int_{0}^{T} \int_{0}^{1}\left\{\psi_{t}(x, t)+\psi_{x x}(x, t)+c \psi(x, t)\right\} y(x, t) d x d t \\
& +\int_{0}^{1} \psi(x, 0) y_{0}(x) d x-\int_{0}^{T} \psi_{x}(1, t) y(1, t) d t .
\end{aligned}
$$

Now, consider a perturbation $\delta \rho$ in the parameter vector $\Theta$, where $\delta$ is a constant of sufficiently small magnitude and $\rho$ is an arbitrary vector. The corresponding perturbation in the state is,

$$
y_{\delta}(x, t)=y(x, t)+\delta\left\langle\nabla_{\Theta} y(x, t), \rho\right\rangle+\mathscr{O}\left(\delta^{2}\right),
$$

and the perturbation in the feedback kernel is,

$$
k_{\delta}(x)=k(x)+\delta\left\langle\nabla_{\Theta} k(x), \rho\right\rangle+\mathscr{O}\left(\delta^{2}\right),
$$

where $\mathscr{O}\left(\delta^{2}\right)$ denotes omitted second-order terms such that $\delta^{-1} \mathscr{O}\left(\delta^{2}\right) \rightarrow 0$ as $\delta \rightarrow 0$. For notational simplicity, we define $\eta(x, t)=\left\langle\nabla_{\Theta} y(x, t), \rho\right\rangle$. Obviously, $\eta(x, 0)=0$, because the initial profile $y_{0}(x)$ is independent of the parameter vector $\Theta$. Based on (24) and (25), the perturbed augmented cost functional takes the following form:

$$
\begin{aligned}
& g_{0}(\Theta+\delta \rho)=\frac{1}{2} \int_{0}^{T} \int_{0}^{1}\{y(x, t)+\delta \eta(x, t)\}^{2} d x d t \\
& \quad+\int_{0}^{T} \int_{0}^{1}\left\{\psi_{t}(x, t)+\psi_{x x}(x, t)\right\}\{y(x, t)+\delta \eta(x, t)\} d x d t \\
& \quad+\int_{0}^{T} \int_{0}^{1} c \psi(x, t)\{y(x, t)+\delta \eta(x, t)\} d x d t \\
& \quad-\int_{0}^{T} \psi_{x}(1, t)\left[\int_{0}^{1} k(x)\{y(x, t)+\delta \eta(x, t)\} d x\right] d t \\
& \quad-\int_{0}^{T} \psi_{x}(1, t)\left[\int_{0}^{1} \delta\left\langle\nabla_{\Theta} k(x), \rho\right\rangle y(x, t) d x\right] d t \\
& \quad+\frac{1}{2} \int_{0}^{1}\left\{k(x)+\delta\left\langle\nabla_{\Theta} k(x), \rho\right\rangle\right\}^{2} d x \\
& \quad+\int_{0}^{1} \psi(x, 0) y_{0}(x) d x+\mathscr{O}\left(\delta^{2}\right) .
\end{aligned}
$$

Taking the derivative of (26) with respect to $\delta$ and setting $\delta=0$ gives

$$
\begin{aligned}
& \left\langle\nabla_{\Theta} g_{0}(\Theta), \rho\right\rangle=\left.\frac{d g_{0}(\Theta+\delta \rho)}{d \delta}\right|_{\delta=0} \\
& =\int_{0}^{T} \int_{0}^{1}\left\{y(x, t)+\psi_{t}(x, t)+\psi_{x x}(x, t)+c \psi(x, t)\right\} \eta(x, t) d x d t \\
& \quad-\int_{0}^{T} \int_{0}^{1} \psi_{x}(1, t) k(x) \eta(x, t) d x d t \\
& \quad-\int_{0}^{T} \int_{0}^{1} \psi_{x}(1, t)\left\langle\nabla_{\Theta} k(x), \rho\right\rangle y(x, t) d x d t \\
& \quad+\int_{0}^{1} k(x)\left\langle\nabla_{\Theta} k(x), \rho\right\rangle d x .
\end{aligned}
$$

Since the perturbation $\rho$ was selected arbitrarily, the theorem follows immediately by setting $\psi(x, t)=v(x, t ; \Theta)$ and taking 


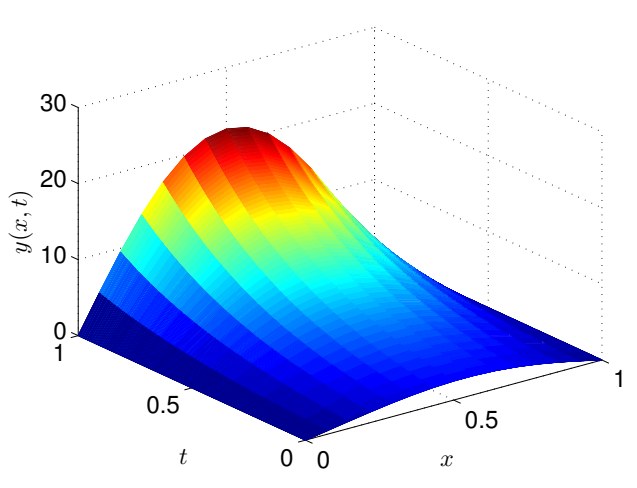

Fig. 2. Uncontrolled open-loop response.

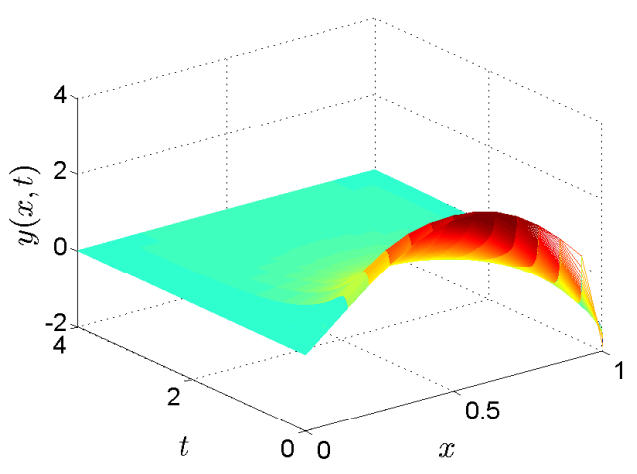

Fig. 3. Optimal closed-loop response.

$\rho$ to be the standard unit basis vectors in $\mathbb{R}^{2}$. This completes the proof.

By combining the gradient formulas in Theorem 3.1 with a standard gradient-based optimization method (such as sequential quadratic programming), Problem 2.2 can be solved efficiently.

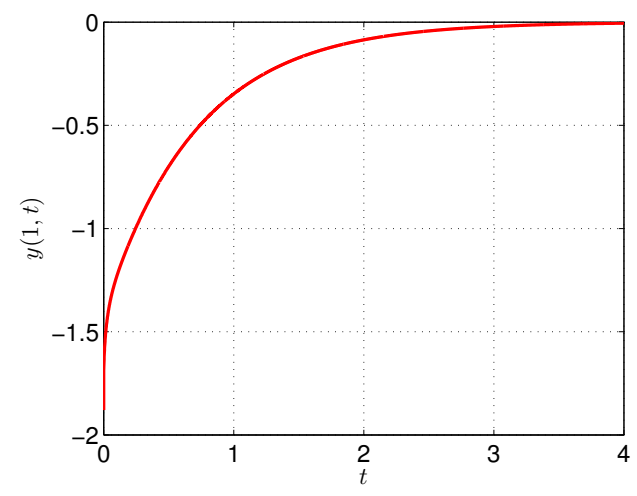

Fig. 4. Optimal boundary control.

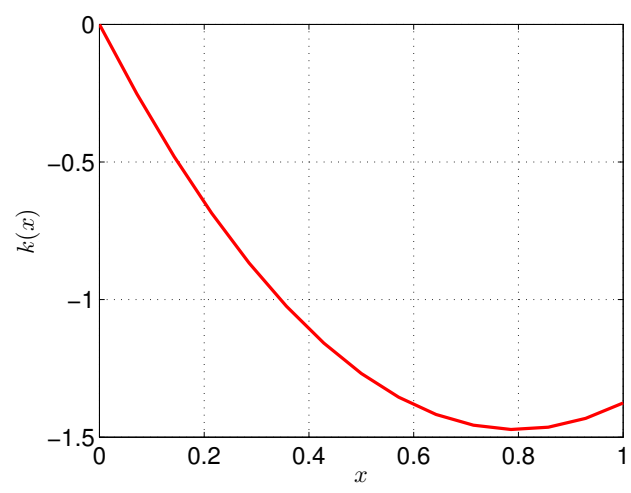

Fig. 5. Optimal kernel function.

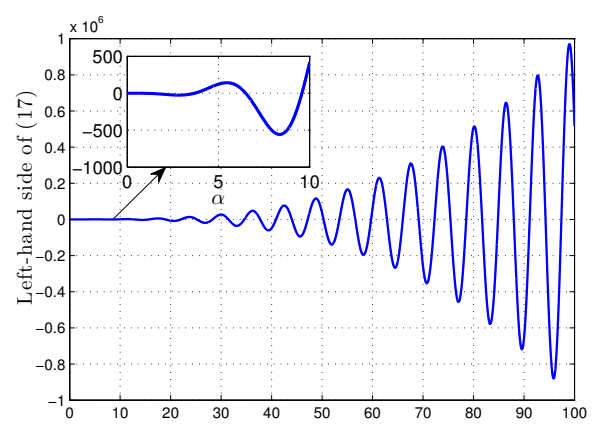

Fig. 6. Equation (17) has an infinite number of positive solutions.

\section{NUMERICAL EXAMPLE}

We consider Problem 2.2 over a time horizon of $[0, T]=$ $[0,4]$. To solve the problem, we wrote a MATLAB program that combines the FMINCON optimization function with the gradient formulas in Theorem 3.1. The state system (3) and the costate system (21) are solved numerically using the finite difference method (with 14 spatial intervals and 5000 temporal intervals). All numerical simulations were performed on a desktop computer with the following configuration: Intel Core i7-2600 3.40GHz CPU, 4.00GB RAM, 64-bit Windows 7 Operating System.

Consider the uncontrolled version of (3) in which $u(t)=0$. In this case, the exact solution is [5]

$$
y(x, t)=2 \sum_{n=1}^{\infty} C_{n} \mathrm{e}^{\left(c-n^{2} \pi^{2}\right) t} \sin (n \pi x) d x,
$$

where $C_{n}$ are the Fourier coefficients defined by

$$
C_{n}=\int_{0}^{1} y_{0}(x) \sin (n \pi x) d x .
$$

The eigenvalues of (27) are $c-n^{2} \pi^{2}, n=1,2, \ldots$ The largest eigenvalue is therefore $c-\pi^{2}$, which indicates that system (3) with $u(t)=0$ is unstable for $c>\pi^{2} \approx 9.8696$.

We choose $c=12$ and $y_{0}(x)=(2+x) \sin (\pi x)$. The corresponding uncontrolled open-loop response (see equation (27)) is shown in Fig. 2. As we can see from Fig. 2, the state 
TABLE I

SOLUTIONS OF EQUATION (17) AND CORRESPONDING SPAN COEFFICIENTS.

\begin{tabular}{crrr}
\hline$n$ & \multicolumn{1}{c}{$\alpha_{n}^{*}$} & \multicolumn{1}{c}{$\alpha_{n}^{*} / \pi$} & \multicolumn{1}{c}{$Y_{n}$} \\
\hline 1 & 3.6934 & 1.0801 & -0.4211 \\
2 & 6.4961 & 2.0678 & 1.5226 \\
3 & 9.5794 & 3.0492 & 1.4629 \\
4 & 12.6751 & 4.0346 & -0.7636 \\
5 & 15.7975 & 5.0285 & 0.4483 \\
6 & 18.9223 & 6.0231 & -0.3458 \\
7 & 22.0544 & 7.0201 & 0.2681 \\
8 & 25.1874 & 8.0173 & 0.9085 \\
9 & 28.3233 & 9.0155 & 1.0726 \\
10 & 31.4597 & 10.0139 & 0.9415 \\
11 & 34.5975 & 11.0127 & 1.0418 \\
12 & 37.7356 & 12.0116 & 0.9709 \\
13 & 40.8745 & 13.0107 & 1.0156 \\
14 & 44.0136 & 14.0099 & 0.9968 \\
15 & 47.1532 & 15.0093 & 0.9908 \\
\hline
\end{tabular}

of the uncontrolled system grows as time increases. For the feedback kernel optimization, we suppose that the lower and upper bounds for the optimization parameters are $a_{i}=-10$ and $b_{i}=10$, respectively. We also choose $\varepsilon=1$ in (19b). Starting from the initial guess $\left(\theta_{1}, \theta_{2}, \alpha\right)=(-1,2,0)$, our program terminates after 29 iterations and 21.0904 seconds. The optimal cost value is $g_{0}=1.2092$ and the optimal solution of Problem 2.2 is $\left(\theta_{1}^{*}, \theta_{2}^{*}, \alpha^{*}\right)=(-3.6977,2.3220,3.6934)$.

The spatial-temporal response of the controlled plant corresponding to $\left(\theta_{1}^{*}, \theta_{2}^{*}\right)$ is shown in Fig. 3. The figure clearly shows that the controlled system (3) with optimized parameters $\left(\theta_{1}^{*}, \theta_{2}^{*}\right)$ is stable. The corresponding optimal boundary control and kernel function are shown in Fig. 4 and Fig. 5, respectively.

Recall from Theorem 2.1 that closed-loop stability is guaranteed if $\alpha^{*}=3.6934$ is the first positive solution of equation (17) and the initial function $y_{0}(x)$ is contained within the linear span of $\left\{\sin \left(\alpha_{n}^{*} x\right)\right\}$, where each $\alpha_{n}^{*}$ is a solution of equation (17) corresponding to $\left(\theta_{1}^{*}, \theta_{2}^{*}\right)$. By viewing a plot of the left-hand side of equation (17), it can be easily verified that $\alpha^{*}$ is indeed the first positive solution; see Fig. 6. To verify the linear span condition, we use FMINCON in MATLAB to minimize (20) for $N=20$. The first 15 positive solutions of (17) corresponding to the optimal parameters $\theta_{1}^{*}=-3.6977$ and $\theta_{2}^{*}=2.3220$ are given in Table I. The optimal span coefficients that minimize (20) are also given. The optimal value of $J$ in $(20)$ is $7.7387 \times 10^{-14}$, which indicates that the span condition holds. Note also from Table I that $\alpha_{n}^{*} / \pi$ converges to an integer as $n \rightarrow \infty$.

\section{CONCLUSIONS}

In this paper, we have introduced a new gradient-based optimization approach for boundary stabilization of parabolic PDE systems. Our new approach involves expressing the boundary controller as an integral state feedback in which a kernel function needs to be designed judiciously. We do not determine the feedback kernel by solving Riccati-type or Klein-Gorden-type PDEs; instead, we approximate the feedback kernel by a quadratic function and then optimize the quadratic's coefficients using dynamic optimization techniques. This preliminary work has also raised several issues that require further investigation: (i) Can the proposed kernel optimization approach be applied to other classes of PDE plant models (i.e., 2D or 3D domains)? (ii) Is it possible to develop methods for minimizing cost functional (7) over an infinite time horizon? These issues will be explored in future work.

\section{REFERENCES}

[1] A. Bensoussan, G. Da Prato, M. C. Delfour and S. K. Mitter, "Representation and Control of Infinite Dimensional Systems", Birkhauser, Boston, 2007.

[2] R. F. Curtain and H. Zwart, "An Introduction to Infinite-dimensional Linear Systems Theory”, Springer, New York, 1995.

[3] N. J. Killingsworth and M. Krstic, "PID tuning using extremum seeking: Online, model-free performance optimization", IEEE Control Systems Magazine, vol. 26, no. 1, pp. 70-79, 2006.

[4] M. Krstic, B. Guo, A. Balogh and A. Smyshlyaev, "Control of a tipforce destabilized shear beam by observer-based boundary feedback", SIAM Journal on Control and Optimization, vol. 47, no. 2, pp. 553-574, 2008.

[5] M. Krstic and A. Smyshlyaev, "Boundary Control of PDEs: A Course on Backstepping Designs", SIAM, Philadelphia, 2008.

[6] B. Li, K. L. Teo, C. Lim and G. Duan, "An optimal PID controller design for nonlinear constrained optimal control problems", Discrete and Continuous Dynamical Systems - Series B, vol. 16, no. 4, pp. 70-79, 2011.

[7] Q. Lin, R. Loxton and K. L. Teo, "Optimal control of nonlinear switched systems: Computational methods and applications", Journal of the Operations Research Society of China, vol. 1, no. 3, pp. 275-311, 2013.

[8] Q. Lin, R. Loxton and K. L. Teo, "The control parameterization method for nonlinear optimal control: A survey", Journal of Industrial and Management Optimization, vol. 10, no. 1, pp. 275-309, 2014.

[9] W. Liu, "Boundary feedback stabilization of an unstable heat equation", SIAM Journal on Control and Optimization, vol. 42, no. 3, pp. 10331043, 2003.

[10] W. Liu, "Elementary Feedback Stabilization of the Linear Reactionconvection-diffusion Equation and the Wave Equation", Springer, Berlin, 2010.

[11] S. J. Moura and H. K. Fathy, "Optimal boundary control of reactiondiffusion partial differential equations via weak variations", Journal of Dynamic Systems, Measurement, and Control, vol. 135, no. 3, 034501(18), 2013.

[12] Z. Ren, C. Xu, Q. Lin and R. Loxton, "A gradient-based kernel optimization approach for parabolic distributed parameter control systems", Pacific Journal of Optimization, accepted.

[13] G. A. Susto and M. Krstic, "Control of PDE-ODE cascades with Neumann interconnections", Journal of the Franklin Institute, vol. 347, no. 1, pp. 284-314, 2010.

[14] K. L. Teo, C. J. Goh and K. H. Wong, "A Unified Computational Approach to Optimal Control Problems", Longman Scientific and Technical, Essex, 1991.

[15] R. Triggiani, "Well-posedness and regularity of boundary feedback parabolic systems", Journal of Differential Equations, vol. 36, no. 3, pp. 347-362, 1980.

[16] R. Vazquez and M. Krstic, "A closed-form feedback controller for stabilization of the linearized 2-D Navier-Stokes Poiseuille system", IEEE Transactions on Automatic Control, vol. 52, no. 12, pp. 22982312, 2007.

[17] R. Vazquez and M. Krstic, "Control of Turbulent and Magnetohydrodynamic Channel Flows: Boundary Stabilization and State Estimation", Springer, Boston, 2008.

[18] J. Xu, D. Huang and S. Pindi, "Optimal tuning of PID parameters using iterative learning approach", SICE Journal of Control, Measurement, and System Integration, vol. 1, no. 3, pp. 143-154, 2008.

[19] C. Xu, E. Schuster, R. Vazquez and M. Krstic, "Stabilization of linearized 2D magnetohydrodynamic channel flow by backstepping boundary control", Systems \& Control Letters, vol. 57, no. 10, pp. 805812, 2008. 


\title{
Output Stabilization of Boundary-controlled Parabolic PDEs via Gradient-based Dynamic Optimization*
}

\author{
Zhigang Ren ${ }^{1}$, Chao $\mathrm{Xu}^{1}$, Qun $\mathrm{Lin}^{2}$, and Ryan Loxton ${ }^{2}$
}

\begin{abstract}
This paper proposes a new control synthesis approach for the stabilization of boundary-controlled parabolic partial differential equations (PDEs). In the proposed approach, the optimal boundary control is expressed in integral state feedback form with quadratic kernel function, where the quadratic's coefficients are decision variables to be optimized. We introduce a system cost functional to penalize both state and kernel magnitude, and then derive the cost functional's gradient in terms of the solution of an auxiliary "costate" PDE. On this basis, the output stabilization problem can be solved using gradient-based optimization techniques such as sequential quadratic programming. The resulting optimal boundary control is guaranteed to yield closed-loop stability under mild conditions. The primary advantage of our new approach is that the costate PDE is in standard form and can be solved easily using the finite difference method. In contrast, the traditional control synthesis approaches for boundary-controlled parabolic PDEs (i.e., the LQ control and backstepping approaches) require solving nonstandard Riccati-type and Klein-Gorden-type PDEs.
\end{abstract}

\section{INTRODUCTION}

Parabolic partial differential equation (PDE) systems are an important type of distributed parameter system (DPS) describing a wide range of natural phenomena, including diffusion, heat transfer, and fusion plasma transport. Over the past few decades, control theory for the parabolic DPS has developed into a mature research topic at the interface of engineering and applied mathematics [1], [2], [10].

The linear quadratic (LQ) control framework is welldefined in infinite dimensional function spaces to deal with the parabolic DPS (e.g., [1], [2]). However, the LQ control framework requires solving Riccati-type differential equations, which are nonlinear parabolic PDEs of dimension one greater than the original parabolic PDE system. For example, to generate an optimal feedback controller for a scalar heat equation, a Riccati PDE defined over a rectangular domain must be solved [11]. Hence, the LQ approach does not actually solve the controller synthesis problem directly, but instead converts it into another problem (i.e., solve a Riccati-type PDE) that is still extremely difficult to solve from a computational point of view.

*This work was supported by the National Natural Science Foundation of China (grants F030119-61104048 and 61320106009) and the National High Technology Research and Development Program of China (grant 2012AA041701)

${ }^{1} \mathrm{Z}$. Ren and C. Xu are with the State Key Laboratory of Industrial Control Technology and the Institute of Cyber-Systems \& Control, Zhejiang University, Hangzhou, China. renzhigang@zju.edu.cn, cxuezju. edu. cn

${ }^{2} \mathrm{Q}$. Lin and R. Loxton are with the Department of Mathematics \& Statistics, Curtin University, Perth, Australia. q. Iin@curtin.edu.au, r.loxtonecurtin.edu. au

Correspondence to: cxu@zju.edu.cn
One of the major advances in PDE control in recent years has been the so-called infinite dimensional Voltera integral feedback, or the backstepping method (e.g., [5], [9]). Instead of Riccati-type PDEs, the backstepping method requires solving the so-called kernel equations-linear Klein-Gordentype PDEs for which the successive approach can be used to obtain explicit solutions. This method was originally developed for the stabilization of one dimensional parabolic DPS and then extended to fluid flows [16], [19], magnetohydrodynamic flows [17], and elastic vibration [4]. In addition, the backstepping method can also be applied to achieve full state feedback stabilization and state estimation of PDE-ODE cascade systems [13].

In this paper, we propose a new framework for control synthesis for boundary-controlled parabolic DPS. This new framework does not require solving Riccati-type or KleinGorden-type PDEs. Instead, it requires solving a so-called "costate" PDE, which is much easier to solve from a computational viewpoint. In fact, many numerical software packages, such as Comsol Multiphysics and MATLAB PDE ToolBox, can be used to generate numerical solutions for the costate PDE. The Riccati PDEs, on the other hand, are usually not in standard form and thus cannot be solved using off-the-shelf software packages. The approach proposed in this paper can be viewed as an extension of optimization-based PID tuning ideas (see [3], [6], [14], [18]) to infinite dimensional systems.

\section{PROBLEM FORMULATION}

\section{A. Feedback Kernel Optimization}

We consider the following parabolic PDE system:

$$
\left\{\begin{array}{l}
y_{t}(x, t)=y_{x x}(x, t)+c y(x, t), \\
y(0, t)=0, \\
y(1, t)=u(t), \\
y(x, 0)=y_{0}(x),
\end{array}\right.
$$

where $c>0$ is a given constant and $u(t)$ is a boundary control. It is well known that the uncontrolled version of system (1) is unstable when the constant $c$ is sufficiently large [5]. According to the LQ control [11] and backstepping synthesis approaches [5], the optimal stabilizing control law takes the following feedback form:

$$
u(t)=\int_{0}^{1} \mathscr{K}(1, \xi) y(\xi, t) d \xi,
$$

where the feedback kernel $\mathscr{K}(1, \xi)$ is obtained by solving either a Riccati-type or a Klein-Gorden-type PDE. By introducing the new notation $k(\xi)=\mathscr{K}(1, \xi)$, we can write the 


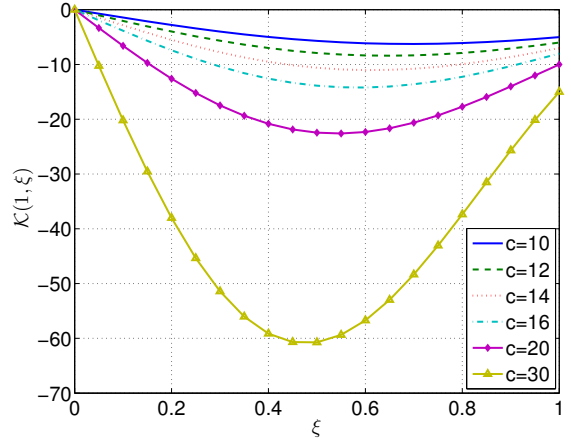

Fig. 1. The feedback kernel (4) for various values of $c$.

feedback control policy (2) in the following form:

$$
u(t)=\int_{0}^{1} k(\xi) y(\xi, t) d \xi .
$$

The corresponding closed-loop system is

$$
\left\{\begin{array}{l}
y_{t}(x, t)=y_{x x}(x, t)+c y(x, t), \\
y(x, 0)=y_{0}(x), \\
y(0, t)=0, \\
y(1, t)=\int_{0}^{1} k(\xi) y(\xi, t) d \xi .
\end{array}\right.
$$

In reference [5], the backstepping method is used to express the optimal feedback kernel as follows:

$$
\mathscr{K}(1, \xi)=-c \xi \frac{I_{1}\left(\sqrt{c\left(1-\xi^{2}\right)}\right)}{\sqrt{c\left(1-\xi^{2}\right)}},
$$

where $I_{1}$ is the first-order modified Bessel function given by

$$
I_{1}(\omega)=\sum_{n=0}^{\infty} \frac{\omega^{2 n+1}}{2^{2 n+1} n !(n+1) !} .
$$

The feedback kernel (4) is plotted in Figure 1 for different values of $c$. Note that its shape is similar to a quadratic function. Note also that $\mathscr{K}(1, \xi)=0$ when $\xi=0$. Accordingly, motivated by the quadratic behavior exhibited in Fig. 1, we express $k(\xi)$ in the following parameterized form:

$$
k(\xi ; \Theta)=\theta_{1} \xi+\theta_{2} \xi^{2},
$$

where $\Theta=\left(\theta_{1}, \theta_{2}\right)^{\top}$ is a parameter vector to be optimized.

Moreover, we assume that the parameters must satisfy the following bound constraints:

$$
a_{1} \leq \theta_{1} \leq b_{1}, \quad a_{2} \leq \theta_{2} \leq b_{2},
$$

where $a_{1}, a_{2}, b_{1}$ and $b_{2}$ are given bounds.

Let $y(x, t ; \Theta)$ denote the solution of the closed-loop system (3) with the parameterized kernel (5). The results in [15] ensure that such a solution exists and is unique. Our goal is to stabilize the closed-loop system with minimal energy input. Accordingly, we consider the following cost functional:

$$
g_{0}(\Theta)=\frac{1}{2} \int_{0}^{T} \int_{0}^{1} y^{2}(x, t ; \Theta) d x d t+\frac{1}{2} \int_{0}^{1} k^{2}(x ; \Theta) d x .
$$

We now state our kernel optimization problem formally as follows.

Problem 2.1: Given the PDE system (3) with the parameterized kernel (5), find an optimal parameter vector $\Theta=$ $\left(\theta_{1}, \theta_{2}\right)^{\top}$ such that the cost functional (7) is minimized subject to the bound constraints (6).

\section{B. Closed-Loop Stability}

Since (7) is a finite-time cost functional, there is no guarantee that the optimized kernel (5) generated by the solution of Problem 2.1 stabilizes the closed-loop system (3) as $t \rightarrow \infty$. Nevertheless, we now show that, by analyzing the solution structure of (3), additional constraints can be added to Problem 2.1 to ensure closed-loop stability.

Using the separation of variables approach, we decompose $y(x, t)$ as follows:

$$
y(x, t)=\mathscr{X}(x) \mathscr{T}(t) .
$$

Substituting (8) into (3a), we obtain

$$
\mathscr{X}(x) \dot{\mathscr{T}}(t)=\mathscr{X}^{\prime \prime}(x) \mathscr{T}(t)+c \mathscr{X}(x) \mathscr{T}(t),
$$

where

$$
\dot{\mathscr{T}}(t)=\frac{d \mathscr{T}(t)}{d t}, \quad \mathscr{X}^{\prime \prime}(x)=\frac{d^{2} \mathscr{X}(x)}{d x^{2}} .
$$

Furthermore, from the boundary conditions (3c) and (3d),

$$
\begin{aligned}
& \mathscr{X}(0) \mathscr{T}(t)=0, \\
& \mathscr{X}(1) \mathscr{T}(t)=\int_{0}^{1} k(\xi ; \Theta) \mathscr{X}(\xi) \mathscr{T}(t) d \xi .
\end{aligned}
$$

Thus, we immediately obtain

$$
\begin{gathered}
\mathscr{X}(0)=0, \\
\mathscr{X}(1)=\int_{0}^{1} k(\xi ; \Theta) \mathscr{X}(\xi) d \xi .
\end{gathered}
$$

Rearranging (9) gives

$$
\frac{\mathscr{X}^{\prime \prime}(x)+c \mathscr{X}(x)}{\mathscr{X}(x)}=\frac{\dot{\mathscr{T}}(t)}{\mathscr{T}(t)}=\sigma,
$$

where $\sigma$ is a constant called the eigenvalue. Clearly,

$$
\mathscr{T}(t)=T_{0} \mathrm{e}^{\sigma t}
$$

where $T_{0}=\mathscr{T}(0)$ is a constant to be determined.

To solve for $\mathscr{X}(x)$, we must consider three cases: (i) $c<\sigma$; (ii) $c=\sigma$; (iii) $c>\sigma$. In cases (i) and (ii), the general solutions of (12) are, respectively,

$$
\mathscr{X}(x)=X_{0} \mathrm{e}^{\sqrt{\sigma-c} x}+X_{1} \mathrm{e}^{-\sqrt{\sigma-c} x},
$$

and

$$
\mathscr{X}(x)=X_{0}+X_{1} x
$$

where $X_{0}$ and $X_{1}$ are constants to be determined from the boundary conditions (10) and (11). Then the corresponding solutions of (3) are

$$
y(x, t)=X_{0} T_{0} \mathrm{e}^{\sqrt{\sigma-c} x+\sigma t}+X_{1} T_{0} \mathrm{e}^{-\sqrt{\sigma-c} x+\sigma t},
$$


and

$$
y(x, t)=X_{0} T_{0} \mathrm{e}^{\sigma t}+X_{1} T_{0} x \mathrm{e}^{\sigma t} .
$$

These solutions are clearly unstable because $0<c \leq \sigma$. Thus, the parameters $\theta_{1}$ and $\theta_{2}$ should be chosen so that the unique solution of (3) satisfies case (iii) instead of cases (i) and (ii).

In case (iii), the general solution of (12) is

$$
\mathscr{X}(x)=X_{0} \cos (\sqrt{c-\sigma} x)+X_{1} \sin (\sqrt{c-\sigma} x),
$$

where $X_{0}$ and $X_{1}$ are constants to be determined from the boundary conditions (10) and (11). Substituting (14) into (10), we obtain

$$
\mathscr{X}(0)=X_{0}=0 .
$$

Hence,

$$
\mathscr{X}(x)=X_{1} \sin (\sqrt{c-\sigma} x) .
$$

To simplify the notation, we introduce a new variable $\alpha=$ $\sqrt{c-\sigma}$. Substituting (15) into condition (11), we have

$$
X_{1} \sin \alpha=X_{1} \int_{0}^{1} \theta_{1} \xi \sin (\alpha \xi) d \xi+X_{1} \int_{0}^{1} \theta_{2} \xi^{2} \sin (\alpha \xi) d \xi
$$

and thus

$$
\sin \alpha=\int_{0}^{1} \theta_{1} \xi \sin (\alpha \xi) d \xi+\int_{0}^{1} \theta_{2} \xi^{2} \sin (\alpha \xi) d \xi .
$$

By evaluating the integrals on the right-hand side, equation (16) can be simplified to obtain

$$
\begin{aligned}
& \left(\theta_{1} \alpha^{2}+\theta_{2} \alpha^{2}-2 \theta_{2}\right) \cos \alpha \\
& \quad+\left(\alpha^{3}-\theta_{1} \alpha-2 \theta_{2} \alpha\right) \sin \alpha+2 \theta_{2}=0 .
\end{aligned}
$$

For any $\alpha$ satisfying (17), there exists a corresponding solution of (12) in the form (15). It can be shown that (17) has an infinite number of positive solutions when $\Theta=\left(\theta_{1}, \theta_{2}\right)^{\top}$ satisfies the following inequality:

$$
\theta_{1}^{2}+\theta_{2}^{2}+2 \theta_{1} \theta_{2}-2 \theta_{1}-4 \theta_{2} \geq 0 .
$$

This is demonstrated numerically in Section IV. A formal proof will be given in a forthcoming journal article [12]. Let $\left\{\alpha_{n}\right\}_{n=1}^{\infty}$ be a sequence of positive solutions of (17). Then the general solution of (12) is

$$
\mathscr{X}(x)=\sum_{n=1}^{\infty} A_{n} \sin \left(\alpha_{n} x\right),
$$

where $A_{n}$ are constants to be determined. The corresponding eigenvalues are

$$
\sigma_{n}=c-\alpha_{n}^{2}, \quad n=1,2,3, \ldots
$$

Hence, using (13),

$$
y(x, t)=\mathscr{X}(x) \mathscr{T}(t)=\sum_{n=1}^{\infty} T_{0} A_{n} \mathrm{e}^{\left(c-\alpha_{n}^{2}\right) t} \sin \left(\alpha_{n} x\right) .
$$

By virtue of (10) and (11), this solution satisfies the boundary conditions (3c) and (3d). The constants $T_{0}$ and $A_{n}$ must be selected appropriately so that the initial condition (3b) is also satisfied. To ensure stability as $t \rightarrow \infty$, each eigenvalue $\sigma_{n}=$ $c-\alpha_{n}^{2}$ in (18) must be negative. Thus, we impose the following constraints on $\Theta=\left(\theta_{1}, \theta_{2}\right)^{\top}$ :

$$
\begin{aligned}
& \theta_{1}^{2}+\theta_{2}^{2}+2 \theta_{1} \theta_{2}-2 \theta_{1}-4 \theta_{2} \geq 0, \\
& c-\alpha^{2} \leq-\varepsilon, \\
& \left(\theta_{1} \alpha^{2}+\theta_{2} \alpha^{2}-2 \theta_{2}\right) \cos \alpha \\
& \quad+\left(\alpha^{3}-\theta_{1} \alpha-2 \theta_{2} \alpha\right) \sin \alpha+2 \theta_{2}=0,
\end{aligned}
$$

where $\varepsilon$ is a given positive parameter and $\alpha$ is the smallest positive solution of (17). Note that $\alpha$ here is treated as an additional optimization variable. Constraint (19a) ensures that there are an infinite number of eigenvalues and thus the solution form (18) is valid. Constraints (19b) and (19c) ensure that the largest eigenvalue is negative, thus guaranteeing solution stability. Adding constraints (19) to Problem 2.1 yields the following modified problem.

Problem 2.2: Given the PDE system (3) with the parameterized kernel (5), choose $\Theta=\left(\theta_{1}, \theta_{2}\right)^{\top}$ and $\alpha$ such that the cost functional (7) is minimized subject to the bound constraints (6) and the nonlinear constraints (19).

The next result is concerned with the stability of the closedloop system corresponding to the optimized kernel from Problem 2.2.

Theorem 2.1: Let $\left(\Theta^{*}, \alpha^{*}\right)$ be an optimal solution of Problem 2.2, where $\alpha^{*}$ is the smallest positive solution of equation $(19 \mathrm{c})$ corresponding to $\Theta^{*}$. Suppose that there exists a sequence $\left\{\alpha_{n}^{*}\right\}_{n=1}^{\infty}$ of positive solutions to equation (19c) corresponding to $\Theta^{*}$ such that $y_{0}(x) \in \operatorname{span}\left\{\sin \left(\alpha_{n}^{*} x\right)\right\}$. Then the closed-loop system (3) corresponding to $\Theta^{*}$ is stable.

Proof: Because of constraint (19a), the solution form (18) with $\alpha_{n}=\alpha_{n}^{*}$ is guaranteed to satisfy (3a), (3c) and (3d). If $y_{0}(x) \in \operatorname{span}\left\{\sin \left(\alpha_{n}^{*} x\right)\right\}$, then there exists constants $Y_{n}, n \geq 1$, such that

$$
y_{0}(x)=\sum_{n=1}^{\infty} Y_{n} \sin \left(\alpha_{n}^{*} x\right) .
$$

Taking $Y_{n}=T_{0} A_{n}$ ensures that (18) with $\alpha_{n}=\alpha_{n}^{*}$ also satisfies the initial conditions ( $3 b)$, and is therefore the unique solution of (3). Since $\alpha^{*}$ is the first positive solution of equation (17), it follows from constraints (19b) and (19c) that for each $n \geq 1$,

$$
c-\left(\alpha_{n}^{*}\right)^{2} \leq c-\left(\alpha^{*}\right)^{2} \leq-\varepsilon<0 .
$$

This shows that all eigenvalues are negative.

Theorem 2.1 requires that the initial function $y_{0}(x)$ be contained within the linear span of sinusoidal functions $\sin \left(\alpha_{n}^{*} x\right)$, where each $\alpha_{n}^{*}$ is a solution of equation (17) corresponding to $\Theta^{*}$. The good thing about this condition is that it can be verified numerically by solving the following optimization problem: choose span coefficients $Y_{n}, 1 \leq n \leq N$, to minimize

$$
J=\int_{0}^{1}\left|y_{0}(x)-\sum_{n=1}^{N} Y_{n} \sin \left(\alpha_{n}^{*} x\right)\right|^{2} d x,
$$

where $N$ is a sufficiently large integer and each $\alpha_{n}^{*}$ is a solution of equation (17) corresponding to the optimal solution of Problem 2.2. If the optimal cost value for this optimization 
problem is sufficiently small, then the span condition in Theorem 2.1 is likely to be satisfied, and therefore closed-loop stability is expected. Based on our computational experience, the span condition in Theorem 2.1 is usually satisfied. In fact, as we show in [12], the solutions $\alpha_{n}^{*}$ of (17) converge to the integer multiples of $\pi$. Thus, it is reasonable to expect that the linear span of $\left\{\sin \left(\alpha_{n}^{*} x\right)\right\}$ is "approximately" the same as the linear span of $\{\sin (n \pi x)\}$, which is known to be a basis for the space of continuous functions defined on $[0,1]$.

\section{NUMERICAL COMPUTATION}

Problem 2.2 is an optimal parameter selection problem with decision parameters $\theta_{1}, \theta_{2}$ and $\alpha$. In principle, such problems can be solved as nonlinear optimization problems using sequential quadratic programming or other nonlinear optimization methods. However, to do this, we need the gradients of the cost functional (7) and the constraint functions (19) with respect to the decision parameters.

Since the constraint functions in (19) are explicit functions of the decision variables, their gradients are easily derived using elementary differentiation. The cost functional (7), on the other hand, is an implicit function of $\Theta$ because it depends on the state trajectory $y(x, t)$. Thus, computing the gradient of (7) is a non-trivial task. We now develop a computational method, analogous to the costate method in the optimal control of ordinary differential equations [7], [8], [14], for computing this gradient.

We define the following costate PDE system:

$$
\left\{\begin{array}{l}
v_{t}(x, t)+v_{x x}(x, t)+c v(x, t) \\
\quad+y(x, t ; \Theta)-k(x ; \Theta) v_{x}(1, t)=0, \\
v(0, t)=v(1, t)=0, \\
v(x, T)=0 .
\end{array}\right.
$$

Let $v(x, t ; \Theta)$ denote the solution of the costate PDE system (21) corresponding to the parameter vector $\Theta$. Then we have the following theorem.

Theorem 3.1: The gradient of the cost functional (7) is given by

$$
\begin{aligned}
& \nabla_{\theta_{1}} g_{0}(\Theta)=-\int_{0}^{T} \int_{0}^{1} x v_{x}(1, t) y(x, t) d x d t+\frac{1}{3} \theta_{1}+\frac{1}{4} \theta_{2}, \\
& \nabla_{\theta_{2}} g_{0}(\Theta)=-\int_{0}^{T} \int_{0}^{1} x^{2} v_{x}(1, t) y(x, t) d x d t+\frac{1}{4} \theta_{1}+\frac{1}{5} \theta_{2},
\end{aligned}
$$

where $y(x, t)=y(x, t ; \Theta)$ and $v_{x}(x, t)=v_{x}(x, t ; \Theta)$.

Proof: For simplicity, we write $y(x, t ; \Theta)$ as $y(x, t)$ and $k(x ; \Theta)$ as $k(x)$. Let $\psi(x, t)$ be an arbitrary function satisfying

$$
\psi(x, T)=0, \quad \psi(0, t)=\psi(1, t)=0 .
$$

Then we can rewrite the cost functional (7) in augmented form as follows:

$$
\begin{aligned}
& g_{0}(\Theta)=\frac{1}{2} \int_{0}^{T} \int_{0}^{1} y^{2}(x, t) d x d t+\frac{1}{2} \int_{0}^{1} k^{2}(x) d x \\
& +\int_{0}^{T} \int_{0}^{1} \psi(x, t)\left\{-y_{t}(x, t)+y_{x x}(x, t)+c y(x, t)\right\} d x d t .
\end{aligned}
$$

Using integration by parts and applying conditions (3b), (3c) and (22), we can simplify the augmented cost functional (23) to obtain

$$
\begin{aligned}
g_{0}(\Theta)= & \frac{1}{2} \int_{0}^{T} \int_{0}^{1} y^{2}(x, t) d x d t+\frac{1}{2} \int_{0}^{1} k^{2}(x) d x \\
& +\int_{0}^{T} \int_{0}^{1}\left\{\psi_{t}(x, t)+\psi_{x x}(x, t)+c \psi(x, t)\right\} y(x, t) d x d t \\
& +\int_{0}^{1} \psi(x, 0) y_{0}(x) d x-\int_{0}^{T} \psi_{x}(1, t) y(1, t) d t .
\end{aligned}
$$

Now, consider a perturbation $\delta \rho$ in the parameter vector $\Theta$, where $\delta$ is a constant of sufficiently small magnitude and $\rho$ is an arbitrary vector. The corresponding perturbation in the state is,

$$
y_{\delta}(x, t)=y(x, t)+\delta\left\langle\nabla_{\Theta} y(x, t), \rho\right\rangle+\mathscr{O}\left(\delta^{2}\right),
$$

and the perturbation in the feedback kernel is,

$$
k_{\delta}(x)=k(x)+\delta\left\langle\nabla_{\Theta} k(x), \rho\right\rangle+\mathscr{O}\left(\delta^{2}\right),
$$

where $\mathscr{O}\left(\delta^{2}\right)$ denotes omitted second-order terms such that $\delta^{-1} \mathscr{O}\left(\delta^{2}\right) \rightarrow 0$ as $\delta \rightarrow 0$. For notational simplicity, we define $\eta(x, t)=\left\langle\nabla_{\Theta} y(x, t), \rho\right\rangle$. Obviously, $\eta(x, 0)=0$, because the initial profile $y_{0}(x)$ is independent of the parameter vector $\Theta$. Based on (24) and (25), the perturbed augmented cost functional takes the following form:

$$
\begin{aligned}
& g_{0}(\Theta+\delta \rho)=\frac{1}{2} \int_{0}^{T} \int_{0}^{1}\{y(x, t)+\delta \eta(x, t)\}^{2} d x d t \\
& \quad+\int_{0}^{T} \int_{0}^{1}\left\{\psi_{t}(x, t)+\psi_{x x}(x, t)\right\}\{y(x, t)+\delta \eta(x, t)\} d x d t \\
& \quad+\int_{0}^{T} \int_{0}^{1} c \psi(x, t)\{y(x, t)+\delta \eta(x, t)\} d x d t \\
& \quad-\int_{0}^{T} \psi_{x}(1, t)\left[\int_{0}^{1} k(x)\{y(x, t)+\delta \eta(x, t)\} d x\right] d t \\
& \quad-\int_{0}^{T} \psi_{x}(1, t)\left[\int_{0}^{1} \delta\left\langle\nabla_{\Theta} k(x), \rho\right\rangle y(x, t) d x\right] d t \\
& \quad+\frac{1}{2} \int_{0}^{1}\left\{k(x)+\delta\left\langle\nabla_{\Theta} k(x), \rho\right\rangle\right\}^{2} d x \\
& \quad+\int_{0}^{1} \psi(x, 0) y_{0}(x) d x+\mathscr{O}\left(\delta^{2}\right) .
\end{aligned}
$$

Taking the derivative of (26) with respect to $\delta$ and setting $\delta=0$ gives

$$
\begin{aligned}
& \left\langle\nabla_{\Theta} g_{0}(\Theta), \rho\right\rangle=\left.\frac{d g_{0}(\Theta+\delta \rho)}{d \delta}\right|_{\delta=0} \\
& =\int_{0}^{T} \int_{0}^{1}\left\{y(x, t)+\psi_{t}(x, t)+\psi_{x x}(x, t)+c \psi(x, t)\right\} \eta(x, t) d x d t \\
& \quad-\int_{0}^{T} \int_{0}^{1} \psi_{x}(1, t) k(x) \eta(x, t) d x d t \\
& \quad-\int_{0}^{T} \int_{0}^{1} \psi_{x}(1, t)\left\langle\nabla_{\Theta} k(x), \rho\right\rangle y(x, t) d x d t \\
& \quad+\int_{0}^{1} k(x)\left\langle\nabla_{\Theta} k(x), \rho\right\rangle d x .
\end{aligned}
$$

Since the perturbation $\rho$ was selected arbitrarily, the theorem follows immediately by setting $\psi(x, t)=v(x, t ; \Theta)$ and taking 


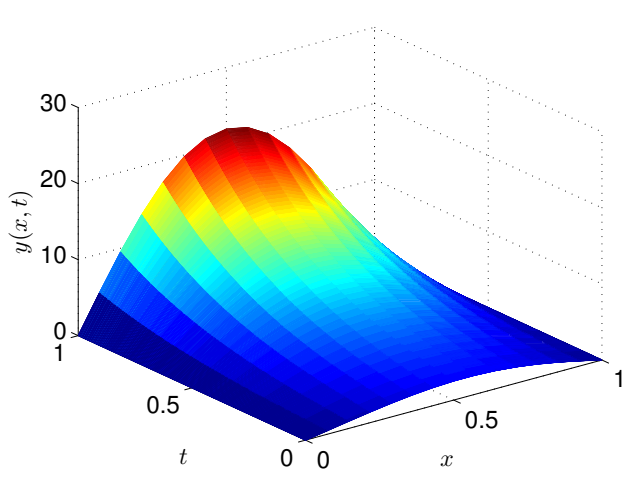

Fig. 2. Uncontrolled open-loop response.

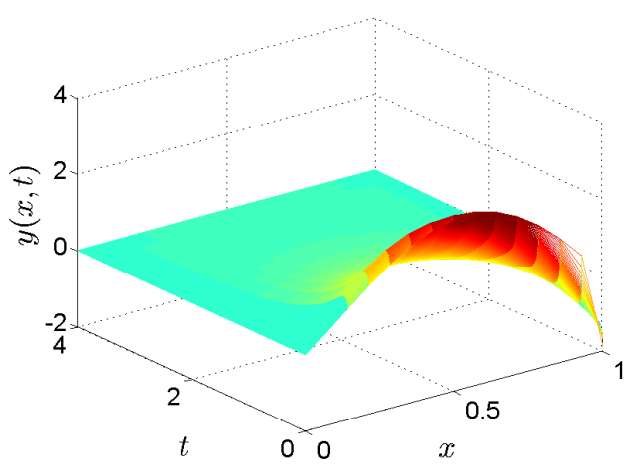

Fig. 3. Optimal closed-loop response.

$\rho$ to be the standard unit basis vectors in $\mathbb{R}^{2}$. This completes the proof.

By combining the gradient formulas in Theorem 3.1 with a standard gradient-based optimization method (such as sequential quadratic programming), Problem 2.2 can be solved efficiently.

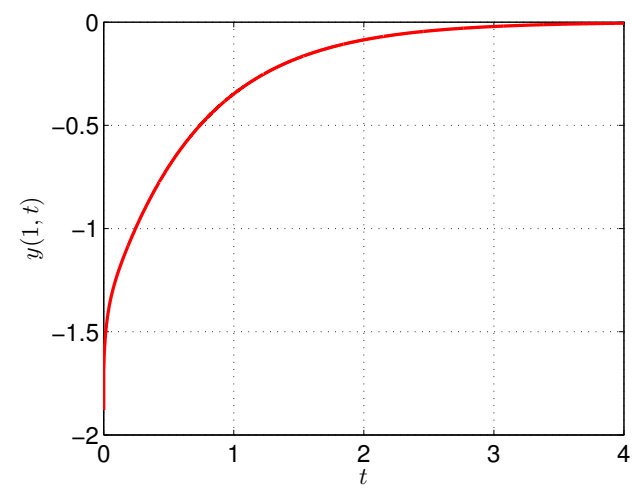

Fig. 4. Optimal boundary control.

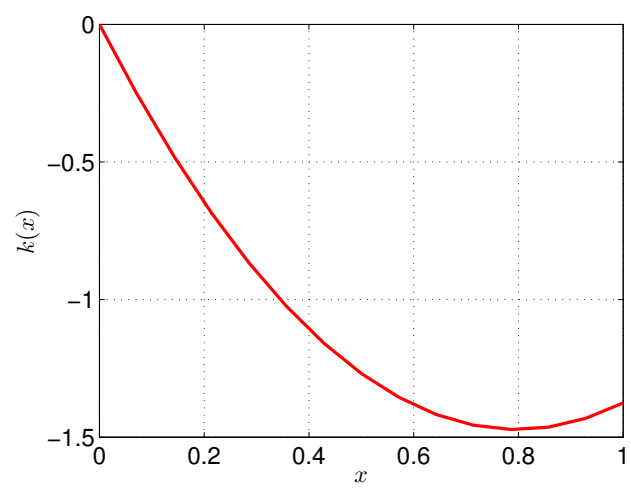

Fig. 5. Optimal kernel function.

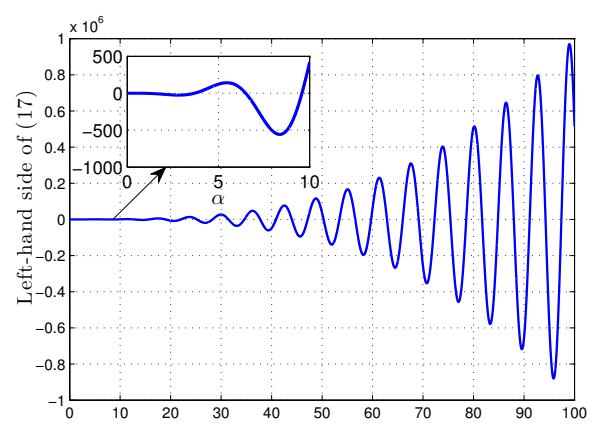

Fig. 6. Equation (17) has an infinite number of positive solutions.

\section{NUMERICAL EXAMPLE}

We consider Problem 2.2 over a time horizon of $[0, T]=$ $[0,4]$. To solve the problem, we wrote a MATLAB program that combines the FMINCON optimization function with the gradient formulas in Theorem 3.1. The state system (3) and the costate system (21) are solved numerically using the finite difference method (with 14 spatial intervals and 5000 temporal intervals). All numerical simulations were performed on a desktop computer with the following configuration: Intel Core i7-2600 3.40GHz CPU, 4.00GB RAM, 64-bit Windows 7 Operating System.

Consider the uncontrolled version of (3) in which $u(t)=0$. In this case, the exact solution is [5]

$$
y(x, t)=2 \sum_{n=1}^{\infty} C_{n} \mathrm{e}^{\left(c-n^{2} \pi^{2}\right) t} \sin (n \pi x) d x,
$$

where $C_{n}$ are the Fourier coefficients defined by

$$
C_{n}=\int_{0}^{1} y_{0}(x) \sin (n \pi x) d x .
$$

The eigenvalues of (27) are $c-n^{2} \pi^{2}, n=1,2, \ldots$ The largest eigenvalue is therefore $c-\pi^{2}$, which indicates that system (3) with $u(t)=0$ is unstable for $c>\pi^{2} \approx 9.8696$.

We choose $c=12$ and $y_{0}(x)=(2+x) \sin (\pi x)$. The corresponding uncontrolled open-loop response (see equation (27)) is shown in Fig. 2. As we can see from Fig. 2, the state 
TABLE I

SOLUTIONS OF EQUATION (17) AND CORRESPONDING SPAN COEFFICIENTS.

\begin{tabular}{crrr}
\hline$n$ & \multicolumn{1}{c}{$\alpha_{n}^{*}$} & \multicolumn{1}{c}{$\alpha_{n}^{*} / \pi$} & \multicolumn{1}{c}{$Y_{n}$} \\
\hline 1 & 3.6934 & 1.0801 & -0.4211 \\
2 & 6.4961 & 2.0678 & 1.5226 \\
3 & 9.5794 & 3.0492 & 1.4629 \\
4 & 12.6751 & 4.0346 & -0.7636 \\
5 & 15.7975 & 5.0285 & 0.4483 \\
6 & 18.9223 & 6.0231 & -0.3458 \\
7 & 22.0544 & 7.0201 & 0.2681 \\
8 & 25.1874 & 8.0173 & 0.9085 \\
9 & 28.3233 & 9.0155 & 1.0726 \\
10 & 31.4597 & 10.0139 & 0.9415 \\
11 & 34.5975 & 11.0127 & 1.0418 \\
12 & 37.7356 & 12.0116 & 0.9709 \\
13 & 40.8745 & 13.0107 & 1.0156 \\
14 & 44.0136 & 14.0099 & 0.9968 \\
15 & 47.1532 & 15.0093 & 0.9908 \\
\hline
\end{tabular}

of the uncontrolled system grows as time increases. For the feedback kernel optimization, we suppose that the lower and upper bounds for the optimization parameters are $a_{i}=-10$ and $b_{i}=10$, respectively. We also choose $\varepsilon=1$ in (19b). Starting from the initial guess $\left(\theta_{1}, \theta_{2}, \alpha\right)=(-1,2,0)$, our program terminates after 29 iterations and 21.0904 seconds. The optimal cost value is $g_{0}=1.2092$ and the optimal solution of Problem 2.2 is $\left(\theta_{1}^{*}, \theta_{2}^{*}, \alpha^{*}\right)=(-3.6977,2.3220,3.6934)$.

The spatial-temporal response of the controlled plant corresponding to $\left(\theta_{1}^{*}, \theta_{2}^{*}\right)$ is shown in Fig. 3. The figure clearly shows that the controlled system (3) with optimized parameters $\left(\theta_{1}^{*}, \theta_{2}^{*}\right)$ is stable. The corresponding optimal boundary control and kernel function are shown in Fig. 4 and Fig. 5, respectively.

Recall from Theorem 2.1 that closed-loop stability is guaranteed if $\alpha^{*}=3.6934$ is the first positive solution of equation (17) and the initial function $y_{0}(x)$ is contained within the linear span of $\left\{\sin \left(\alpha_{n}^{*} x\right)\right\}$, where each $\alpha_{n}^{*}$ is a solution of equation (17) corresponding to $\left(\theta_{1}^{*}, \theta_{2}^{*}\right)$. By viewing a plot of the left-hand side of equation (17), it can be easily verified that $\alpha^{*}$ is indeed the first positive solution; see Fig. 6. To verify the linear span condition, we use FMINCON in MATLAB to minimize (20) for $N=20$. The first 15 positive solutions of (17) corresponding to the optimal parameters $\theta_{1}^{*}=-3.6977$ and $\theta_{2}^{*}=2.3220$ are given in Table I. The optimal span coefficients that minimize (20) are also given. The optimal value of $J$ in $(20)$ is $7.7387 \times 10^{-14}$, which indicates that the span condition holds. Note also from Table I that $\alpha_{n}^{*} / \pi$ converges to an integer as $n \rightarrow \infty$.

\section{CONCLUSIONS}

In this paper, we have introduced a new gradient-based optimization approach for boundary stabilization of parabolic PDE systems. Our new approach involves expressing the boundary controller as an integral state feedback in which a kernel function needs to be designed judiciously. We do not determine the feedback kernel by solving Riccati-type or Klein-Gorden-type PDEs; instead, we approximate the feedback kernel by a quadratic function and then optimize the quadratic's coefficients using dynamic optimization techniques. This preliminary work has also raised several issues that require further investigation: (i) Can the proposed kernel optimization approach be applied to other classes of PDE plant models (i.e., 2D or 3D domains)? (ii) Is it possible to develop methods for minimizing cost functional (7) over an infinite time horizon? These issues will be explored in future work.

\section{REFERENCES}

[1] A. Bensoussan, G. Da Prato, M. C. Delfour and S. K. Mitter, "Representation and Control of Infinite Dimensional Systems", Birkhauser, Boston, 2007.

[2] R. F. Curtain and H. Zwart, "An Introduction to Infinite-dimensional Linear Systems Theory”, Springer, New York, 1995.

[3] N. J. Killingsworth and M. Krstic, "PID tuning using extremum seeking: Online, model-free performance optimization", IEEE Control Systems Magazine, vol. 26, no. 1, pp. 70-79, 2006.

[4] M. Krstic, B. Guo, A. Balogh and A. Smyshlyaev, "Control of a tipforce destabilized shear beam by observer-based boundary feedback", SIAM Journal on Control and Optimization, vol. 47, no. 2, pp. 553-574, 2008.

[5] M. Krstic and A. Smyshlyaev, "Boundary Control of PDEs: A Course on Backstepping Designs", SIAM, Philadelphia, 2008.

[6] B. Li, K. L. Teo, C. Lim and G. Duan, "An optimal PID controller design for nonlinear constrained optimal control problems", Discrete and Continuous Dynamical Systems - Series B, vol. 16, no. 4, pp. 70-79, 2011.

[7] Q. Lin, R. Loxton and K. L. Teo, "Optimal control of nonlinear switched systems: Computational methods and applications", Journal of the Operations Research Society of China, vol. 1, no. 3, pp. 275-311, 2013.

[8] Q. Lin, R. Loxton and K. L. Teo, "The control parameterization method for nonlinear optimal control: A survey", Journal of Industrial and Management Optimization, vol. 10, no. 1, pp. 275-309, 2014.

[9] W. Liu, "Boundary feedback stabilization of an unstable heat equation", SIAM Journal on Control and Optimization, vol. 42, no. 3, pp. 10331043, 2003.

[10] W. Liu, "Elementary Feedback Stabilization of the Linear Reactionconvection-diffusion Equation and the Wave Equation", Springer, Berlin, 2010.

[11] S. J. Moura and H. K. Fathy, "Optimal boundary control of reactiondiffusion partial differential equations via weak variations", Journal of Dynamic Systems, Measurement, and Control, vol. 135, no. 3, 034501(18), 2013.

[12] Z. Ren, C. Xu, Q. Lin and R. Loxton, "A gradient-based kernel optimization approach for parabolic distributed parameter control systems", Pacific Journal of Optimization, accepted.

[13] G. A. Susto and M. Krstic, "Control of PDE-ODE cascades with Neumann interconnections", Journal of the Franklin Institute, vol. 347, no. 1, pp. 284-314, 2010.

[14] K. L. Teo, C. J. Goh and K. H. Wong, "A Unified Computational Approach to Optimal Control Problems", Longman Scientific and Technical, Essex, 1991.

[15] R. Triggiani, "Well-posedness and regularity of boundary feedback parabolic systems", Journal of Differential Equations, vol. 36, no. 3, pp. 347-362, 1980.

[16] R. Vazquez and M. Krstic, "A closed-form feedback controller for stabilization of the linearized 2-D Navier-Stokes Poiseuille system", IEEE Transactions on Automatic Control, vol. 52, no. 12, pp. 22982312, 2007.

[17] R. Vazquez and M. Krstic, "Control of Turbulent and Magnetohydrodynamic Channel Flows: Boundary Stabilization and State Estimation", Springer, Boston, 2008.

[18] J. Xu, D. Huang and S. Pindi, "Optimal tuning of PID parameters using iterative learning approach", SICE Journal of Control, Measurement, and System Integration, vol. 1, no. 3, pp. 143-154, 2008.

[19] C. Xu, E. Schuster, R. Vazquez and M. Krstic, "Stabilization of linearized 2D magnetohydrodynamic channel flow by backstepping boundary control", Systems \& Control Letters, vol. 57, no. 10, pp. 805812, 2008. 